\title{
Celecoxib, a selective COX-2 inhibitor, markedly reduced the severity of tamoxifen-induced adenomyosis in a murine model
}

\author{
ZHIXING JIN ${ }^{1-3}$, XIAOYI WU ${ }^{2}$, HAIOU LIU ${ }^{1-3}$ and CONGJIAN XU ${ }^{1-3}$ \\ ${ }^{1}$ Department of Obstetrics and Gynecology, Shanghai Medical College of Fudan University; \\ ${ }^{2}$ Obstetrics and Gynecology Hospital of Fudan University; ${ }^{3}$ Shanghai Key Laboratory of Female Reproductive \\ Endocrine Related Diseases, Fudan University, Shanghai 200011, P.R. China
}

Received February 28, 2019; Accepted December 5, 2019

DOI: $10.3892 / \mathrm{etm} .2020 .8580$

\begin{abstract}
The aim of the present study was to evaluate the effects of the selective cyclooxygenase (COX)-2 inhibitor celecoxib on the development of uterine adenomyosis in mice. ICR neonatal mice were first exposed to tamoxifen to establish a mouse model of adenomyosis. Following 60 days of celecoxib treatment, pathological formation of adenomyosis lesions and the depth of myometrial infiltration were evaluated using hematoxylin and eosin staining. To examine thermal pain modulation in mice, a hotplate test was conducted every 15 days from postnatal day 30 onwards. Immunohistochemistry was performed to assess the expression of aromatase P450, $\mathrm{N}$-cadherin, E-cadherin, COX-2 and cluster of differentiation 31 , whereas the levels of estrogen were analyzed in uterine tissue homogenates using ELISA. Masson trichrome staining was performed to assess the extent of fibrosis in the uterus. Celecoxib treatment significantly inhibited the depth of infiltration into the myometrium, resulting in significantly reduced disease severity. Treatment with high doses of celecoxib significantly prolonged thermal response latency. Following celecoxib treatment, the expression of E-cadherin was significantly increased whereas the expression of $\mathrm{N}$-cadherin was significantly decreased. Concomitantly, the extent of fibrosis was also reduced following celecoxib treatment. Uterine tissue homogenates isolated from mice treated with both high and low doses of celecoxib exhibited lower concentrations of estrogen and decreased expression of aromatase P450. These observations suggest that celecoxib reduces adenomyosis severity by suppressing estrogen production in the uterus, reversing epithelial-mesenchymal transition and relieving fibrosis. Taken together, the results of the present study support
\end{abstract}

Correspondence to: Professor Haiou Liu or Professor Congjian $\mathrm{Xu}$, Obstetrics and Gynecology Hospital of Fudan University, 128 Shenyang Road, Shanghai 200011, P.R. China

E-mail: liuhaiou@fudan.edu.cn

E-mail: xucongjian@fudan.edu.cn

Key words: celecoxib, adenomyosis, epithelial-mesenchymal transition, estrogen, angiogenesis, fibrosis the potential use of celecoxib, a selective COX-2 inhibitor, for the treatment of adenomyosis.

\section{Introduction}

Adenomyosis is an estrogen-dependent gynecological condition that is characterized by pelvic pain, aberrant uterine bleeding and infertility (1). However, the clinical presentation of adenomyosis remains ambiguous. Although magnetic resonance imaging has improved the diagnostic accuracy of adenomyosis, this condition remains asymptomatic with a prevalence of 21 to $33 \%$ in women of reproductive age (2). Despite the prevalence and severity of symptoms associated with adenomyosis, little is known about the pathogenesis and etiology of this condition. To date, two main theories have been proposed to explain the origin of adenomyosis: i) Invagination of the endometrial basalis as a result of the activation of tissue injury and repair mechanism; and ii) metaplasia of displaced embryonic pluripotent Mullerian remnants or differentiation of adult stem cells (3). At present, the main method for treating symptomatic adenomyosis is to relieve symptoms and improve fertility whilst minimizing side effects (4). Although there have been a number randomized, double-blind clinical studies exploring the possible pharmacological interventions for adenomyosis (5-7), no such strategy is currently available for adenomyosis. In addition, no specific guidelines exist for the optimal management of this condition.

Similar to endometriosis, adenomyosis is closely associated with inflammation and angiogenesis $(8,9)$, to which the prostaglandin cascade initiated by cyclooxygenases (COXs) may serve an important role. There are two types of COXs, COX-1 and COX-2, which can be differentially inhibited by a large number of nonsteroidal anti-inflammatory drugs (NSAIDs). NSAIDs are non-hormonal compounds that are commonly used as symptomatic treatment for dysmenorrhea and heavy bleeding associated with adenomyosis $(10,11)$. Findings from recent studies have suggested that NSAIDs are also capable of inhibiting angiogenesis, proliferation, the expression of aromatase P450 and epithelial-mesenchymal transition (EMT) in numerous cancer models, such as colorectal cancer, prostate cancer and human glioblastoma endothelial cells, highlighting their potentially novel properties (12-14). Additionally, the long-term use of NSAIDs has been proposed as a therapeutic 
strategy for a number of malignancies, including prostate, colon and breast cancer (15-18).

The NSAIDs that are currently available can be classified into two main families: Non-selective NSAIDs (nsNSAIDs) and selective COX-2 inhibitors (coxibs). According to their half-lives, nsNSAIDs can be classified further into two groups: Those with a short half-life ( $<6 \mathrm{~h})$, including ibuprofen, and those with longer half-lives, including naproxen (19). Ibuprofen and naproxen are commonly used for dysmenorrhea in patients with adenomyosis. However, the therapeutic application of nsNSAIDs is limited by gastrointestinal and renal complications as a result of long-term COX-1 inhibition (20). In contrast, coxibs selectively act on the COX-2 isoenzyme which maintain the anti-inflammatory properties of traditional NSAIDs without effecting the activity of the COX-1 isoenzyme (21).

Therefore, the aim of the present study is to examine the efficacy of celecoxib, a potent COX-2 inhibitor, on the establishment of adenomyosis lesions in a murine model. In addition, the extent of EMT, vascularization and estrogen production in uterine tissue homogenates were evaluated.

\section{Materials and methods}

Preparation of animals. All experiments were performed under the guidelines of the National Research Council's Guide for the Care and Use of Laboratory Animals (22), and were approved by the Institutional Experimental Animals Review Board of Shanghai Obstetrics and Gynecology Hospital of Fudan University (Shanghai, China). A total of 12, 19-day pregnant ICR mice, weighing 40-50 g, were purchased from Laboratory Animal Center of the Shanghai Institutes of Biological Sciences. Each dam and her pups were housed in the same cage under a controlled environment with 70-80\% humidity at $22^{\circ} \mathrm{C}$ with $12 \mathrm{~h}$ light/dark cycles and had access to chow and freshwater ad libitum.

Mouse model of adenomyosis and animal treatment. A total of 70 female neonatal mice were born from 12 pregnant ICR mice, where all the male neonatal progeny were sacrificed by cervical dislocation. The neonates were randomly divided into 7 groups: i) Control $(\mathrm{n}=10)$; ii) adenomyosis model (ADE) $(\mathrm{n}=10)$; iii) low-dose celecoxib (celecoxib low) $(\mathrm{n}=10)$; iv) high-dose celecoxib (celecoxib high) $(\mathrm{n}=10)$; v) naproxen $(\mathrm{n}=10)$; vi) aspirin $(\mathrm{n}=10)$; and vii) ibuprofen $(\mathrm{n}=10)$. The mice in the control group were dosed with the solvent [peanut oil/lecithin/condensed milk mixture $(10: 1: 15 \mathrm{v} / \mathrm{v})]$ only, while those in the ADE group were orally dosed with tamoxifen to induce adenomyosis and the mice in the other groups were orally dosed with tamoxifen and the corresponding drugs. Negative control group was obtained from the control group which was incubated in PBS instead of primary antibodies for the exclusion of non-specific staining.

The female neonatal mice in the ADE and drug treatment groups were treated orally with $1 \mathrm{mg} / \mathrm{kg}$ tamoxifen (Shanghai Fudan Forward Pharmaceutical Co., Ltd.) suspended in a peanut oil/lecithin/condensed milk mixture $(10: 1: 15 \mathrm{v} / \mathrm{v})$ at a dose volume of $5 \mu \mathrm{l} / \mathrm{g}$ body weight, once daily on days 2 to 5 after birth (day of birth=day 1), as reported previously (23). From day 6 after birth, mice were administered orally with either a low dose of celecoxib (50 mg/kg; Pfizer, Inc.), a high dose of celecoxib (100 mg/kg; Pfizer, Inc.), naproxen (20 mg/kg; cat. no. N8280; Sigma-Aldrich; Merck KGaA), aspirin $(160 \mathrm{mg} / \mathrm{kg}$; cat. no. A2093; Sigma-Aldrich; Merck KGaA) or ibuprofen (30 mg/kg; cat. no. I4883; Sigma-Aldrich; Merck KGaA). The mice in the control and ADE groups were orally administered similarly with corresponding quantities of the vehicle. All mice were weaned and separated from the dams upon reaching 3 weeks of age, where the dams were sacrificed using cervical dislocation. From aged 30 days, all mice were subjected to a hotplate test every 15 days until day 60 . Uterine samples from the mice in the seven groups aforementioned were harvested at 60 days of age.

Hotplate test procedure. The hotplate test was performed using a commercially available Hot Plate Analgesia Meter (BME-480; Chinese Academy of Medical Sciences) as described previously (24). The surface of the plate was heated to and maintained at a constant temperature of $50.0 \pm 0.1^{\circ} \mathrm{C}$ as measured using a built-in digital thermometer. A plastic cylinder of $20 \mathrm{~cm}$ in diameter and $18 \mathrm{~cm}$ in height was placed on the hotplate. All mice were allowed to acclimatize under a controlled environment at $22-24^{\circ} \mathrm{C}$ with $70-80 \%$ humidity in the testing room for $10 \mathrm{~min}$ prior to test commencement. The latency response to thermal stimuli was defined as the time (sec) elapsed from the moment the mouse was placed inside the cylinder to the moment it licked its hind paws. Each mouse was tested once per session. The latency was calculated as the mean between two readings recorded between $24 \mathrm{~h}$ intervals.

Specimen collection. After treatment for 60 days, all mice were anaesthetized and perfused with warm $\left(37^{\circ} \mathrm{C}\right)$ saline followed by a warm solution of $4 \%$ paraformaldehyde plus $0.2 \%$ picric acid dissolved in $0.1 \mathrm{M}$ phosphate buffer, which served as the fixative, via the ascending aorta. The uteri were then excised, where half of the samples were immediately fixed in $4 \%$ paraformaldehyde at room temperature for $48 \mathrm{~h}$ and then embedded in paraffin for hematoxylin and eosin (H\&E) or immunohistochemical staining. All remaining uterine tissue samples were placed immediately in liquid nitrogen for subsequent determination of estrogen concentration in uterine tissue homogenates.

Immunohistochemistry. Each paraffin-embedded tissue block was subjected to serial $3 \mathrm{~mm}$ sectioning for H\&E staining for the assessment of lesion formation or subsequent immunohistochemical staining of E-cadherin (cat. no. ab76055; 1:100; Abcam), N-cadherin (cat. no. ab18203; 1:1,000; Abcam), CD31 (cat. no. ab28364; 1:50; Abcam), aromatase P450 (cat. no. ab28146; 1:50; Abcam) or COX-2 (cat. no. ab15191; 1:100; Abcam). Briefly, paraffin-embedded slides were deparaffinized, rehydrated and washed in PBS (pH 7.4) three times for 15 min each. Then the slices were subjected to antigen retrieval with citric acid buffer $(\mathrm{pH}$ 6.0) at $98^{\circ} \mathrm{C}$ for a total of $30 \mathrm{~min}$. Next they were treated with $3 \%$ hydrogen peroxide (Beyotime Institute of Biotechnology) and blocked with $10 \%$ goat serum (Beyotime Institute of Biotechnology) for $1 \mathrm{~h}$ at room temperature and incubated with the aforementioned primary antibodies or PBS (negative group) overnight at $4^{\circ} \mathrm{C}$. After washing with PBS (pH 
7.4) three times for $15 \mathrm{~min}$ each, the sections were incubated with the horseradish peroxidase-conjugated secondary antibody (cat. no. ab6728; 1:2,000; Abcam) at room temperature for $60 \mathrm{~min}$. Subsequently, the sections were washed again with PBS and incubated with $0.01 \%$ 3,3'-diaminobenzidine tetrahydrochloride (Beyotime Institute of Biotechnology) for $2 \mathrm{~min}$ at room temperature. The sections were then washed thoroughly in PBS three times for 5 min each, stained in hematoxylin for $20 \mathrm{sec}$ at room temperature, dehydrated in an ascending absolute alcohol gradient, washed with xylene and mounted in synthetic resin for microscopic examination with a light microscope.

Quantification of immunoreactivity was performed using Image Pro-Plus 6.0 (Media Cybernetics, Inc.) software, as described previously (25). Vascular microvessels were quantified with CD31, in the stromal compartment of the uteri, where the CD31-labeled microvessel density was quantified under x200 magnification. The average number was calculated from 5 randomly selected images per tissue section, as previously described (26).

Estradiol concentration in uterine tissue homogenates. The mouse uterine tissues were homogenized in PBS (pH 7.2-7.4) and centrifuged at $5,000 \mathrm{x} \mathrm{g}$ at $4^{\circ} \mathrm{C}$ before supernatants were collected. Estradiol (mouse) ELISA kit (K3830-100, BioVision, Inc.) was used to determine the concentration of estradiol in mouse uteri, according to manufacturer's protocol. Each assay was performed in triplicate.

Masson's trichrome staining. Masson's trichrome staining was used for the detection of collagen fibers in tissue samples. Tissue sections were deparaffinized in xylene, rehydrated in a descending graded alcohol series and incubated in Bouin's solution at $37^{\circ} \mathrm{C}$ for $2 \mathrm{~h}$. The Bouin's solution consisted of $75 \mathrm{ml}$ saturated picric acid, $25 \mathrm{ml} 10 \%$ formalin solution $(\mathrm{v} / \mathrm{v})$ and $5 \mathrm{ml}$ acetic acid. Tissue sections were then stained using the Masson's Trichrome Staining kit (Baso Diagnostics, Inc.) according to manufacturer's protocols. The areas of the collagen fiber layer stained in blue were obtained by light microscope under a x200 magnification and quantified using Image Pro-Plus 6.0 software (Media Cybernetics, Inc.).

Western blot analysis. The endometrial adenocarcinoma cell line Ishikawa cells were purchased from the National Infrastructure of Cell Line Resources and cultured at $37^{\circ} \mathrm{C}$ in DMEM/F12 medium (Gibco; Thermo Fisher Scientific, Inc.) supplemented with $10 \%$ FBS. Ishikawa cells were separately treated with DMSO, $1 \mu \mathrm{M} \beta$-Estradiol (cat. no. E8875; Sigma-Aldrich; Merck KGaA), $0.1 \mu \mathrm{M} \beta$-Estradiol, $50 \mu \mathrm{M}$ celecoxib and $50 \mu \mathrm{M}$ celecoxib $+1 \mu \mathrm{M} \beta$-Estradiol at $37^{\circ} \mathrm{C}$ for $48 \mathrm{~h}$, then cell proteins were extracted and western blot analysis was performed as previously described (20). Primary antibodies used included E-cadherin (cat. no. ab76055; 1:1,000; Abcam), anti-GAPDH (cat. no. ab181602; 1:3,000; Abcam), pan-cytokeratin (cat. no. ab7753; 1:1,000; Abcam) and vimentin (cat. no. ab193555; 1:1,000; Abcam). The secondary antibodies that were used included goat anti-rabbit IgG (cat. no. ab6721; 1:1,000; Abcam) and goat anti-mouse IgG (cat. no. ab6728; 1:1,000; Abcam). The densities of the bands were quantified using Quantity One Analysis software v4.62 (Bio-Rad Laboratories, Inc.) and normalized against those of GAPDH.

Statistical analysis. SPSS18.0 software (SPSS, Inc.) was used for all statistical analyses. Comparisons of means from multiple groups were performed using a one-way ANOVA followed by Dunnett's post hoc test. Data is presented as the mean \pm SEM. $\mathrm{P}<0.05$ was considered to indicate a statistically significant difference.

\section{Results}

Infiltration of myometrium by the endometrium following celecoxib treatment. The depth of ectopic endometrial infiltration into the myometrium was evaluated using the criteria previously described by Bird et al (27): Grade 0, complete absence of any ectopic endometrium in the myometrium; grade 1, penetration of the ectopic endometrium into the superficial myometrium; grade 2, penetration into the mid myometrium; and grade 3 , penetration beyond the mid myometrium (Fig. 1A; representative images are from each group at 60 days post-birth to demonstrate the detailed criteria that was used in the present study). As demonstrated in Fig. 1B, compared with the ADE group, mice in the control group exhibited significantly decreased infiltration. Mice treated with either low or high dose celecoxib, naproxen or aspirin demonstrated significantly reduced myometrial infiltration compared with untreated mice in the ADE group. There was no significant difference in the depth of myometrium infiltration between mice treated with ibuprofen and untreated mice from the ADE group. Compared with mice treated with nsNSAIDs, naproxen, aspirin and ibuprofen, mice treated with the selective COX-2 inhibitor celecoxib exhibited less myometrium infiltration.

Effect of celecoxib treatment on hotplate response latency following adenomyosis establishment. The hotplate test is a commonly used method for measuring nociception and evaluating response threshold to thermal stimuli in rodents (28). In the present study, all mice were subjected to hotplate testing every 15 days from 30 days after birth (Fig. 1C). Hotplate response latency in mice from the ADE group was significantly decreased compared with those in the control group. On days 30 and 45 after birth, treatment with high-dose celecoxib and aspirin significantly prolonged the response latency compared with the ADE group. However, a significantly prolonged response latency was not observed in the aspirin treatment group at 60 days after birth (Fig. 1C).

Celecoxib treatment inhibits the expression of $\mathrm{COX}-2$ in the uterus following adenomyosis establishment. Under physiological conditions, COX-1 is expressed in almost all tissues and cells and it serves a protective role in the gastrointestinal tract (29). In contrast, COX-2 is not universally expressed in the majority of normal tissues, but can be rapidly induced following stimulation by proinflammatory factors, lipopolysaccharides or growth factors; in turn leading to the occurrence and development of a variety of diseases like atherogenesis, human macula densa and so on (30).

As reported by previous studies, COX-2 expression increases significantly during the pathogenesis of adenomyosis (31-33). 
A

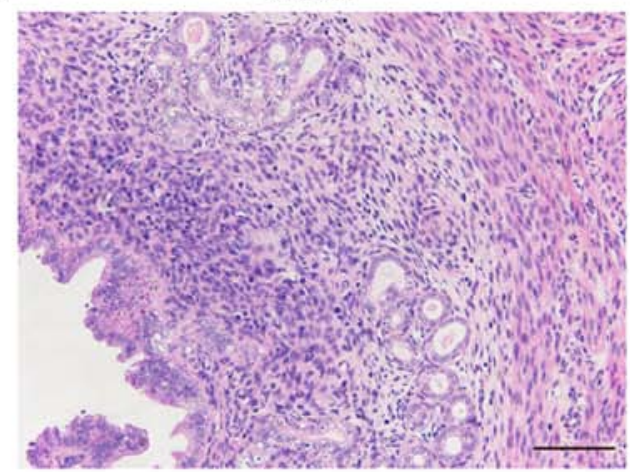

Grade 2

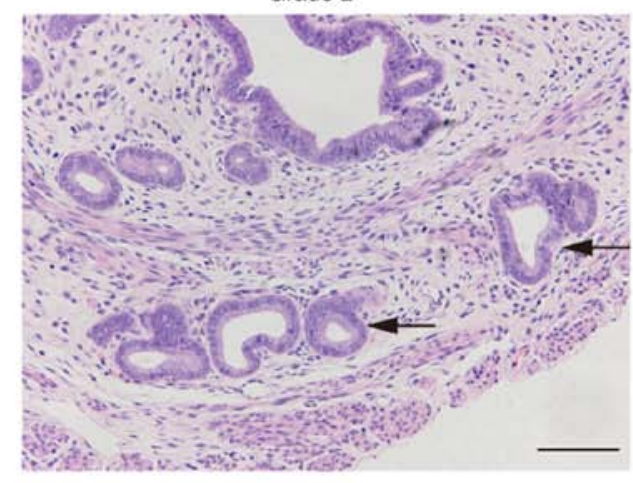

Grade 1

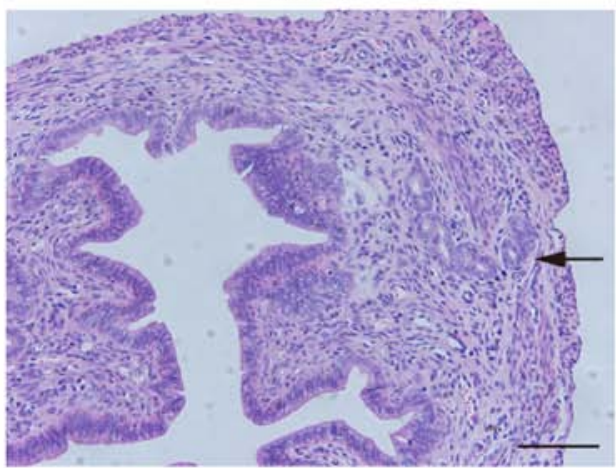

Grade 3

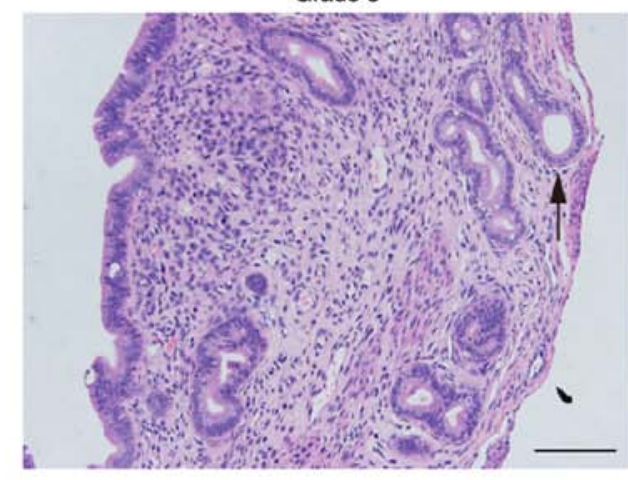

Control

w ADE

曰Celecoxib low

떼 Celecoxib high

[a. Naproxen

Aspirin

E巴I Ibuprofen

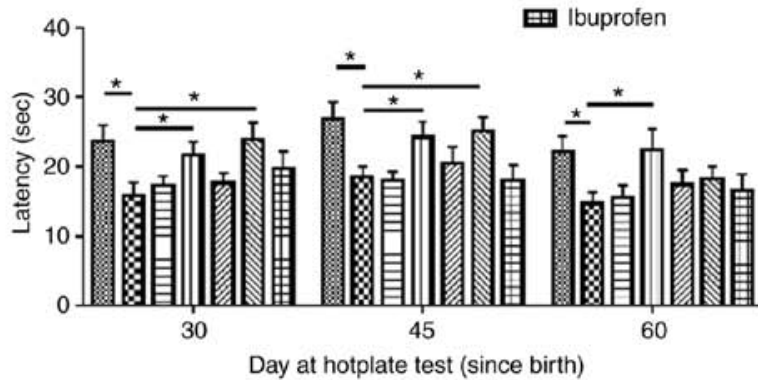

Figure 1. Celecoxib markedly reduced the depth of endometrial infiltration into the myometrium in a tamoxifen-induced adenomyosis mouse model. (A) Classification of adenomyosis progression in an experimentally induced ICR mouse model, with each of the four grades illustrated by the corresponding representative images from all treatment groups at 60 days after birth to show the detailed criteria that was used. The arrows are used to demonstrate where the ectopic endometrium invaded into the myometrium. Scale bars, $100 \mu \mathrm{m}$. (B) Quantified grades of myometrial infiltration by the endometrium following treatment with nsNSAIDs and celecoxib at day 60 after birth. (C) Hotplate latency test of mice subjected to nsNSAIDs or celecoxib treatment at the indicated times. ${ }^{* * *} \mathrm{P}<0.001,{ }^{* *} \mathrm{P}<0.01$ and ${ }^{*} \mathrm{P}<0.05$ vs. ADE. nsNSAIDs, non-selective nonsteroidal anti-inflammatory drugs; ADE, adenomyosis.

Therefore, immunohistochemical staining was used to assess the expression of COX-2 following NSAIDs and celecoxib treatment. As indicated in Fig. 2, compared with the control group, mice in the ADE group exhibited significantly increased COX-2 expression following adenomyosis induction. Treatment with high and low dose celecoxib and nsNSAIDs significantly reduced the expression of COX-2 compared with the ADE group.

Celecoxib treatment reduces estrogen production in the uterus following adenomyosis establishment. Aromatase P450 is considered to be the rate-limiting enzyme during estrogen synthesis in the uterus (34). Therefore, in the present study the expression of aromatase $\mathrm{P} 450$ was evaluated in uterine tissues. As presented in Fig. 3A and B, aromatase P450 expression was predominantly localized in the cytoplasm of glandular epithelial cells in the eutopic endometrium and lesions within the myometrium. The expression level of aromatase P450 in the uterus was significantly higher in mice from the ADE group compared with the control group. Following treatment with low-dose celecoxib, high-dose celecoxib or naproxen, a significant decrease in aromatase P450 was observed in the uterus compared with the untreated ADE group. However, no significant difference was observed following ibuprofen or aspirin treatment. 

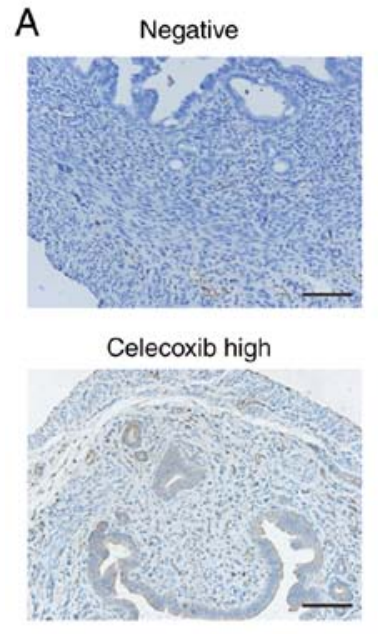

Control

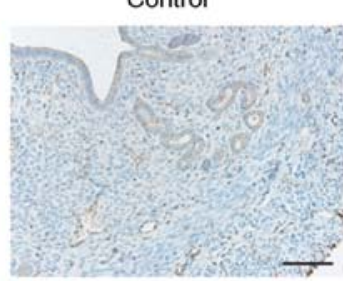

Naproxen

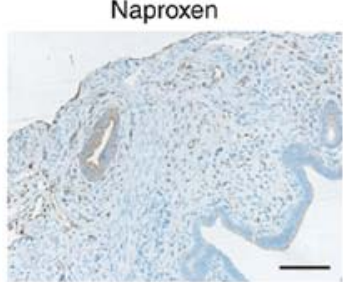

ADE

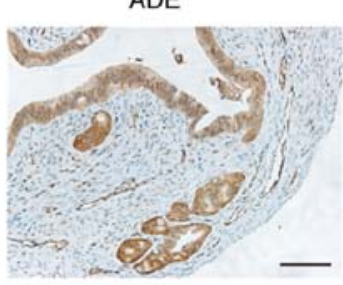

Aspirin

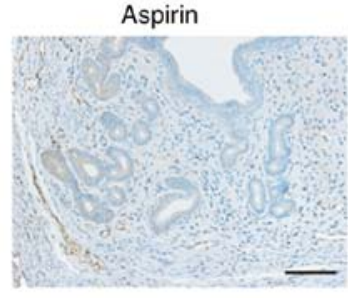

Celecoxib low

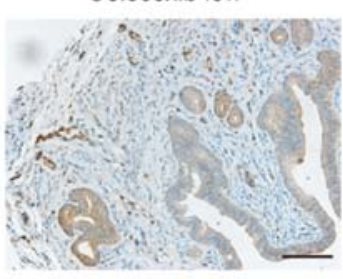

Ibuprofen

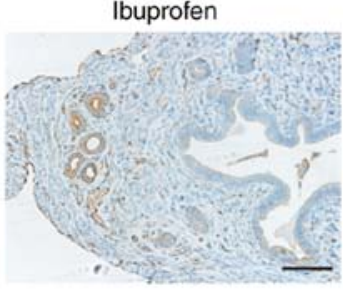

\section{B}

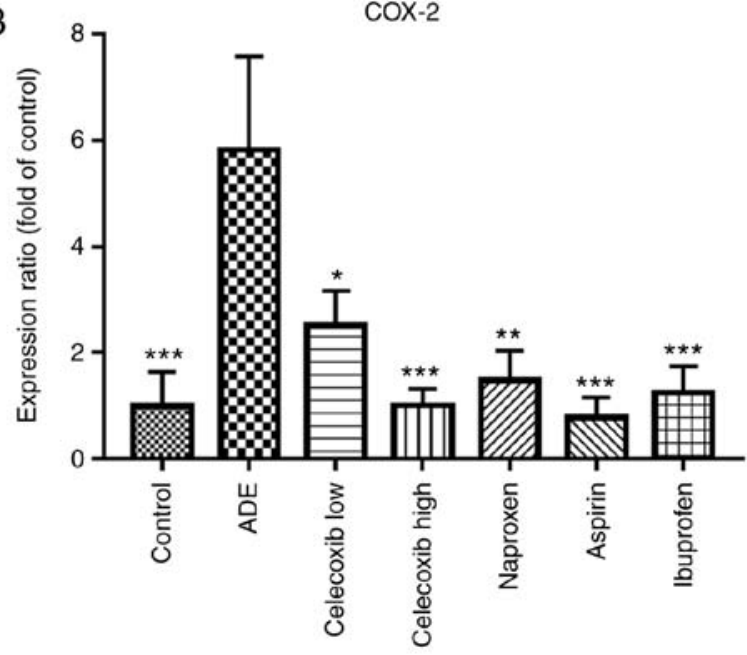

Figure 2. Celecoxib significantly reduces the expression of COX-2 in the uterus, which was evaluated by immunohistochemical staining following 60 days of treatment. (A) Representative immunohistochemical staining images of COX-2 in specimens from mice treated with non-selective nonsteroidal anti-inflammatory drugs or celecoxib. Scale bars, $100 \mu \mathrm{m}$. (B) Quantification of immunohistochemical COX-2 staining in uteri from the seven groups. ${ }^{* * *} \mathrm{P}<0.001,{ }^{* * *} \mathrm{P}<0.01$ and ${ }^{*} \mathrm{P}<0.05$ vs. ADE. COX-2, cyclooxygenase-2; ADE, adenomyosis.

ELISA was subsequently performed to determine the levels of estradiol (E2) in uterine tissue homogenates. The levels of E2 in uterine tissue homogenates of untreated mice from the ADE group were revealed to be significantly higher compared with the control group (Fig. 3C). Following celecoxib and nsNSAID treatment, the levels of E2 in the uterine tissue homogenates were significantly lower compared with those of untreated mice from the ADE group (Fig. 3C).

Celecoxib treatment reverses EMT following the establishment of adenomyosis. Immunohistochemistry was used to assess whether the expression of $\mathrm{N}$-cadherin and E-cadherin was affected by celecoxib or NSAIDs treatment. As indicated in Fig. 4, E-cadherin was mainly expressed in the membrane of epithelial cells, where its levels were significantly reduced following adenomyosis induction. With the exception of naproxen, treatment with ibuprofen, aspirin and celecoxib significantly increased E-cadherin expression compared with the ADE group. In contrast, the opposite effects were observed regarding $\mathrm{N}$-cadherin expression, which was significantly increased following adenomyosis induction, but was reduced following ibuprofen, aspirin or high celecoxib treatment, while low celecoxib and naproxen didn't affect the N-cadherin expression significantly (Fig. 5).

Progression of adenomyosis to fibrosis is repressed by celecoxib treatment. Masson's trichrome staining was performed to evaluate fibrosis in the mouse uterine tissues following treatments with NSAIDs and celecoxib. Portions of the stromal cells, muscle fibers or epithelial cells were stained red, whilst the collagen fibers were stained blue.

Collagen staining was observed to be almost negative in uterine tissues isolated from mice in the control group, whereas advanced adenomyotic lesions obtained from mice from the ADE group contained more collagen fibers (Fig. 6A and B). In addition, the extent of fibrosis, as indicated by the expression of collagen, was indicated to be significantly reduced by treatment with ibuprofen, aspirin, low-dose and high-dose celecoxib compared with mice from the ADE group (Fig. 6).

Effect of NSAIDs treatment on uterine vascular density following adenomyosis establishment. To determine vascular density, blood vessels in the uterine tissues were stained using the anti-CD31 antibody, where the number of vessels per 
A
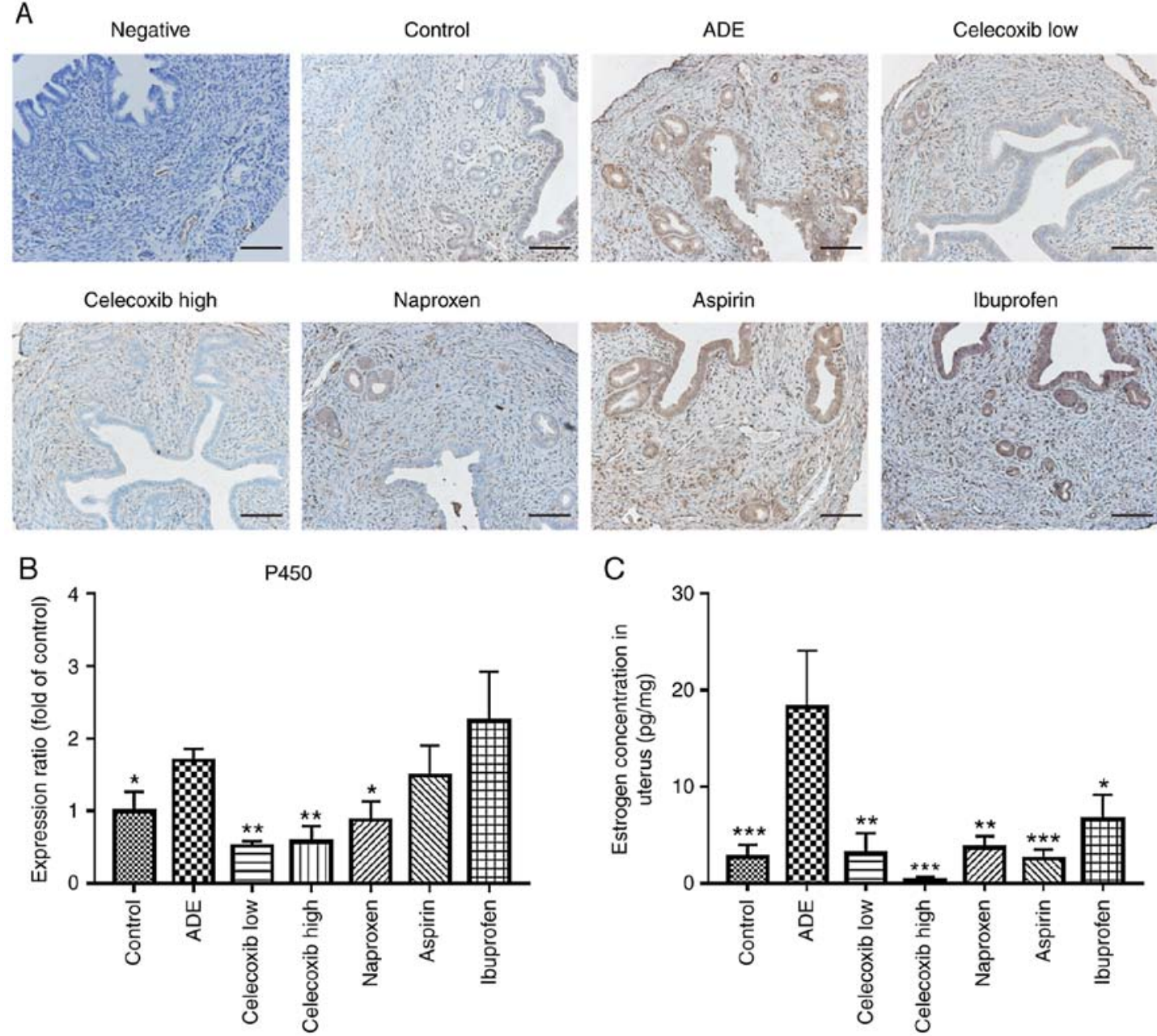

Figure 3. Celecoxib significantly inhibits estrogen production in the uterus. (A) Representative immunohistochemical staining images of aromatase P450 in specimens from mice treated with nsNSAIDs or celecoxib. The arrow is used to identify the ectopic endometrium into the myometrium. Scale bars, $100 \mu \mathrm{m}$. (B) Quantification of immunohistochemical aromatase P450 staining in uteri from the seven groups. (C) Estradiol levels in uterine tissue homogenates following nsNSAIDs or celecoxib treatment for 60 days, as measured by ELISA. ${ }^{* * *} \mathrm{P}<0.001,{ }^{* *} \mathrm{P}<0.01$ and ${ }^{*} \mathrm{P}<0.05$ vs. ADE. nsNSAIDs, non-selective nonsteroidal anti-inflammatory drugs; ADE, adenomyosis.

area was subsequently quantified (Fig. 7). Compared with the control group, there was a statistically significant increase in vessel density detected in the ADE group. Although a statistically significant reduction was observed in vessel density in the uterine tissues following treatment with naproxen, no significant differences were observed in the ibuprofen, aspirin, low-dose or high-dose celecoxib-treated groups compared with the ADE group.

Celecoxib inhibits EMT in Ishikawa cells following estradiol treatment. Previous reports have demonstrated that $\mathrm{E}_{2}$ can induce EMT in endometrial epithelial cells during adenomyosis (35-37). To demonstrate if it is a direct effect of celecoxib on epithelialization, Ishikawa cells were treated with DMSO, $0.1 \mu \mathrm{M} \mathrm{E}_{2}, 1 \mu \mathrm{M} \mathrm{E}_{2}, 50 \mu \mathrm{M}$ celecoxib or $50 \mu \mathrm{M}$ celecoxib $+1 \mu \mathrm{M} \mathrm{E}_{2}$ for $48 \mathrm{~h}$, following which western blot analysis was performed to determine the expression of proteins associated with EMT.

Following treatment with $\mathrm{E}_{2}$, the expression of epithelial markers E-cadherin and pan-cytokeratin was observed to be significantly downregulated in Ishikawa cells, which was accompanied by the concomitant upregulation of the mesenchymal marker vimentin (Fig. 8). Treatment with Celecoxib reversed the aforementioned effects (Fig. 8).

\section{Discussion}

Adenomyosis is a major cause of pelvic pain and infertility in women. Due to the lack of understanding in the pathogenesis of adenomyosis, treatment options that are current available remain inadequate, which mainly focus upon pain relief and improving fertility (38). A number of studies have previously reported that in addition to containing anti-inflammatory and analgesic properties, NSAIDs also serve important roles in a variety of biological functions (39-41). In the present study, the potential therapeutic effects of NSAIDs were evaluated in a murine model of tamoxifen-induced adenomyosis, and the results indicated that NSAIDs and the COX-2 selective inhibitor celecoxib markedly reduced the depth of endometrial infiltration into the myometrium, with celecoxib demonstrating the greatest efficacy. In addition, high-dose celecoxib treatment prolonged the thermal response latency, suggesting that COX-2 inhibition reduced pain sensitivity in mice with adenomyosis. 


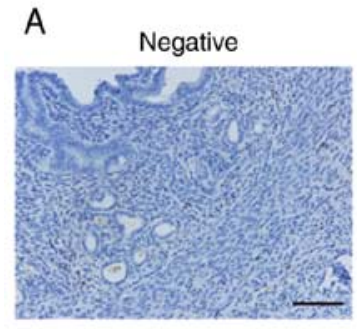

Celecoxib high

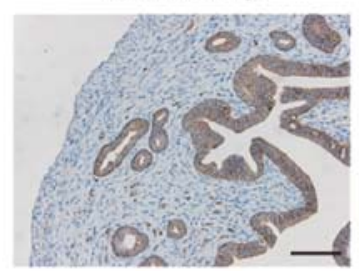

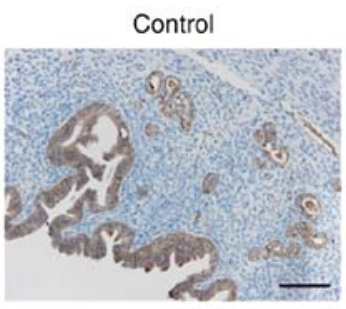

Naproxen

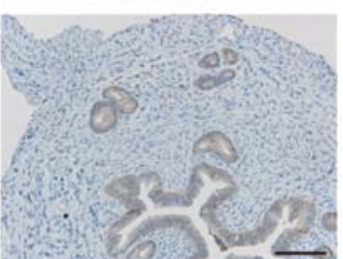

ADE

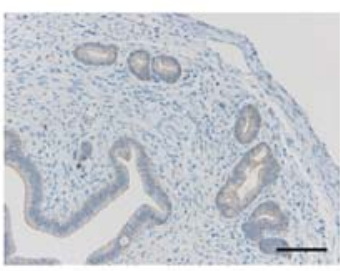

Aspirin

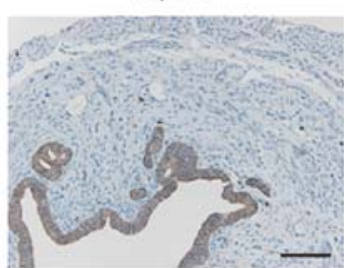

B

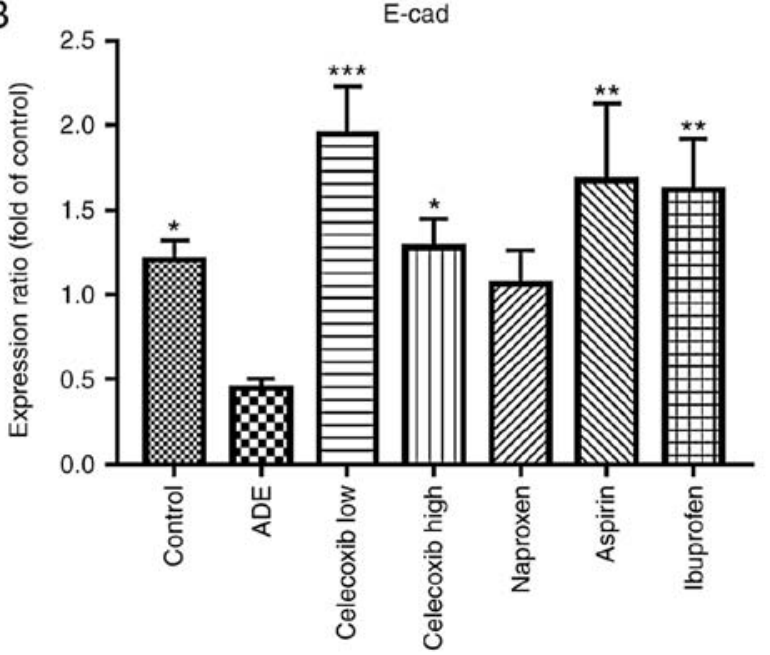

Figure 4. Celecoxib significantly increased the expression of E-cadherin in uteri from mice with adenomyosis. (A) Representative immunohistochemical staining images of E-cadherin in specimens from mice subjected to non-selective nonsteroidal anti-inflammatory drugs or celecoxib treatment for 60 days. Scale bars, $100 \mu \mathrm{m}$. (B) Quantification of immunohistochemical E-cadherin staining in uteri from the seven groups. ${ }^{* * *} \mathrm{P}<0.001,{ }^{* *} \mathrm{P}<0.01$ and ${ }^{*} \mathrm{P}<0.05 \mathrm{vs}$. ADE. E-cad, E-cadherin; ADE, adenomyosis.

Steroid hormones are closely associated with the etiology of adenomyosis. In particular, hyperestrogenism as a result of aberrant local paracrine activity in the eutopic and ectopic endometrium may serve as a preliminary risk factor for the initiation of this disease (42). This notion is supported by findings from a previous study that elevated levels of E2 were frequently observed in the menstrual blood of women with adenomyosis compared with peripheral blood levels (43). Hyperestrogenism is suggested to result from increased local aromatization of androgens and reduced local estrogen metabolism in the eutopic and ectopic endometrium (44). Notably, Kitawaki et al (45) reported that the expression aromatase cytochrome $\mathrm{P} 450$, which is a rate-limiting enzyme in steroidogenesis, was not observed in the endometrium of healthy uteri. In contrast, aromatase cytochrome $\mathrm{P} 450$ was present in the eutopic endometrium of patients with adenomyosis, which promoted estrogen biosynthesis and increased estrogen bioavailability due to the local aromatization of circulating androgens into E2.

In the present study, E2 and P450 expression levels were increased in the uterus of mice in the ADE group. Following celecoxib and naproxen treatment, the expression of P450 in the uterus was significantly lower compared with untreated ADE mice, which may explain the reduced E2 concentration in the corresponding uterine tissue homogenates. Although the uterine levels of E2 were reduced in the aspirin and ibuprofen group, the expression of P450 was not significantly decreased, suggesting that additional factors may be involved, including the expression of 17- $\beta$ hydroxysteroid dehydrogenase type 2 enzyme, which has been previously revealed to contribute to E2 degradation (46).

EMT is characterized by the loss of cell-cell adhesions between the normally stationary polarized epithelial cells, which transform into the highly motile mesenchymal cells. It is a biological process involved in embryological development, tissue repair and cancer cell migration and invasion (47). Consistent with previous studies (48), reduced E-cadherin expression and increased $\mathrm{N}$-cadherin expression were detected in the epithelial cells of adenomyotic lesions compared with normal endometrium in the present study, suggesting that invasiveness and progression of adenomyotic lesions could be regulated by EMT. Following celecoxib treatment, the expression of E-cadherin in epithelial cells was significantly 


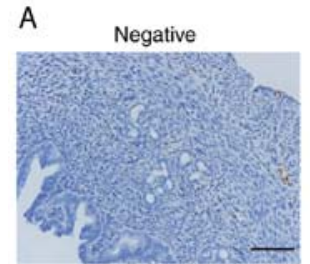

Celecoxib high

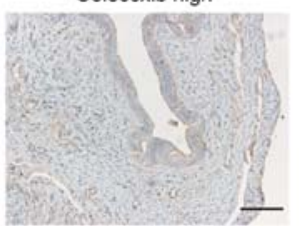

Control

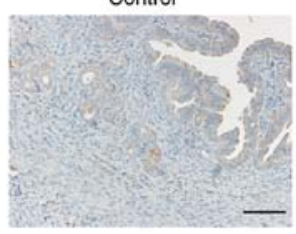

Naproxen

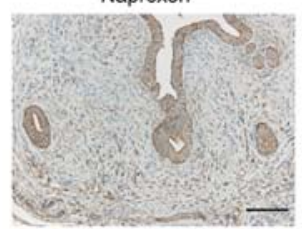

ADE

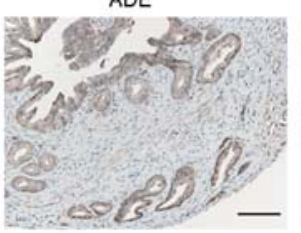

Aspirin

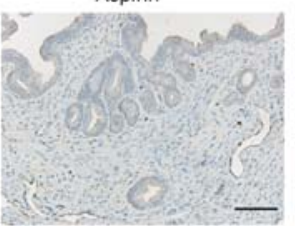

Celecoxib low

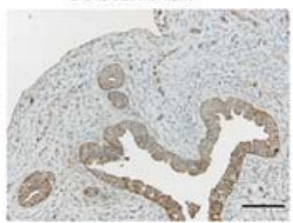

Ibuprofen

B

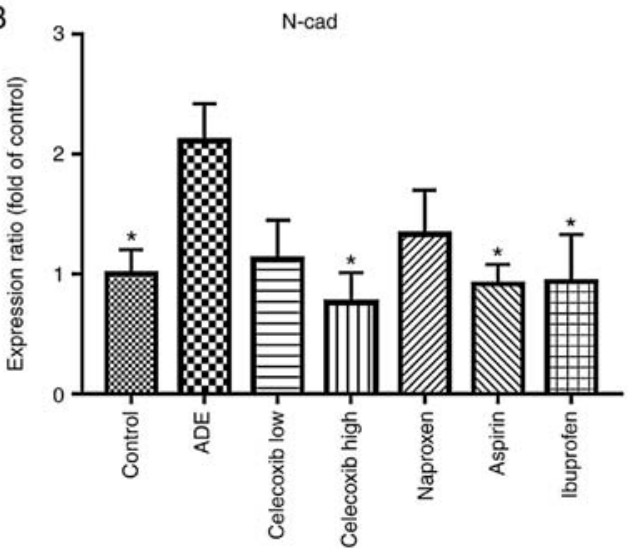

Figure 5. Celecoxib significantly reduces the expression of $\mathrm{N}$-cadherin in uteri from mice with adenomyosis, as evaluated by immunoreactivity staining after of treatment. (A) Representative immunohistochemical staining images of $\mathrm{N}$-cadherin in specimens from mice treated with non-selective nonsteroidal anti-inflammatory drugs or celecoxib for 60 days. Scale bars, $100 \mu \mathrm{m}$. (B) Quantification of immunohistochemical N-cadherin staining in uteri from the seven groups. "P<0.05 vs. ADE. N-cad, N-cadherin; ADE, adenomyosis.

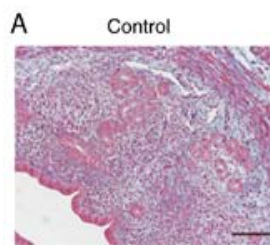

Naproxen

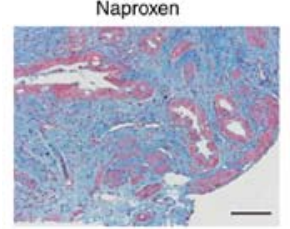

ADE

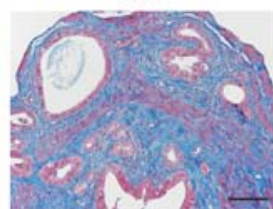

Aspirin

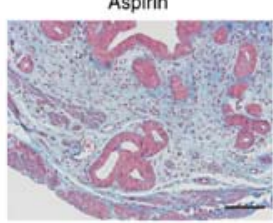

B
Celecoxib low

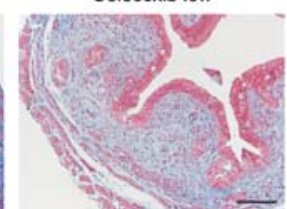

Ibuprofen

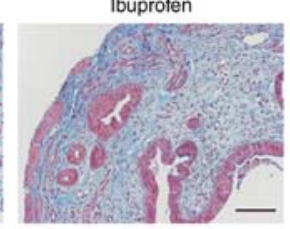

Celecoxib high
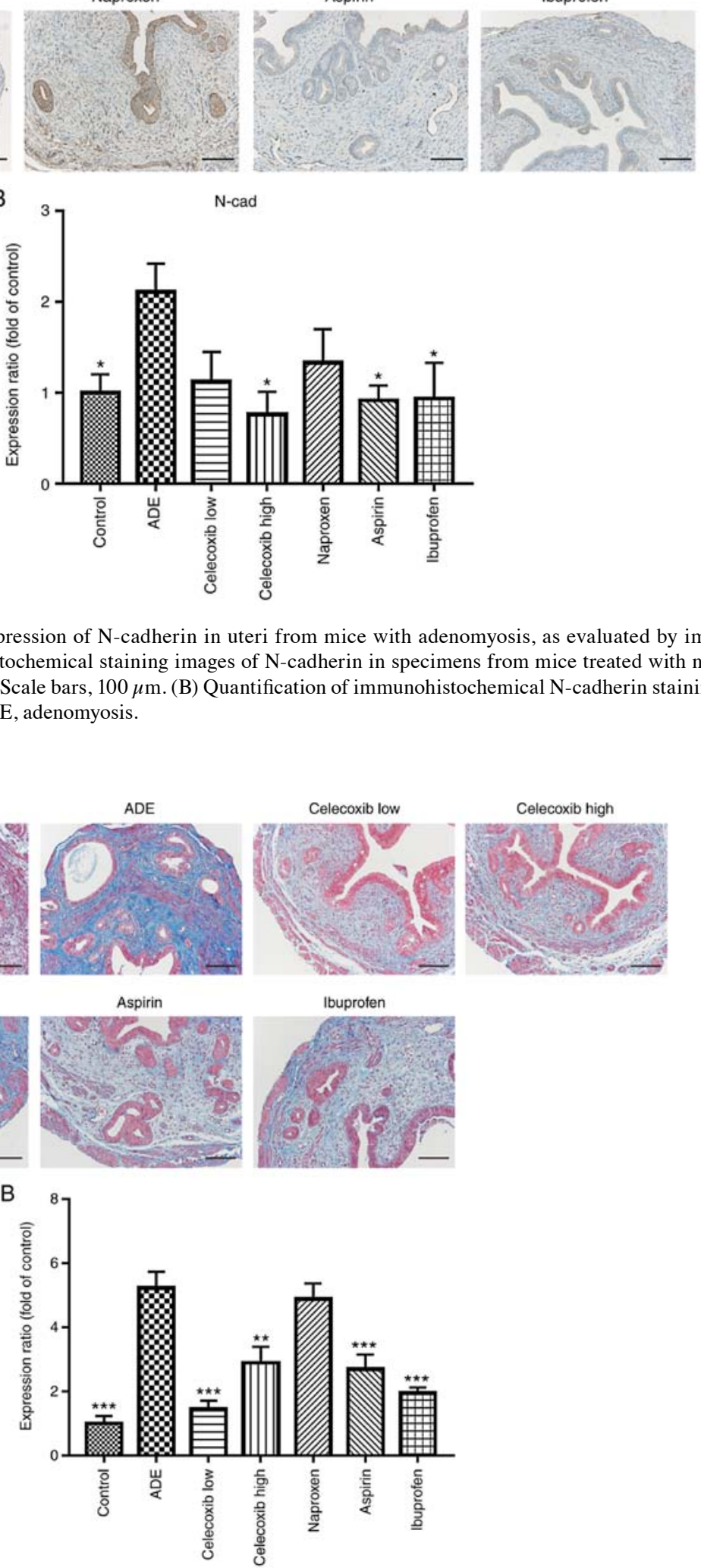

Figure 6. Progression of adenomyosis to fibrosis in mouse uteri is inhibited by celecoxib treatment. (A) Representative images of Masson's trichrome staining in specimens from mice treated with nsNSAIDs or celecoxib. Scale bars, $100 \mu \mathrm{m}$. (B) Quantification of collagen fibers staining, shown in blue, following nsNSAIDs or celecoxib treatment. ${ }^{* * *} \mathrm{P}<0.001$ and ${ }^{* *} \mathrm{P}<0.01$ vs. ADE. nsNSAIDs, non-selective nonsteroidal anti-inflammatory drugs; ADE, adenomyosis. 
A

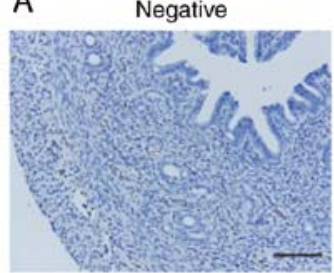

Celecoxib high

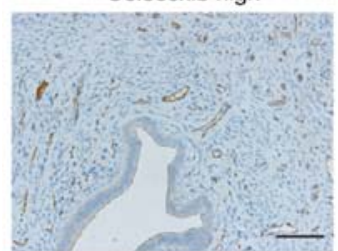

Control

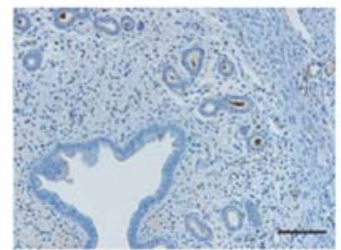

Naproxen

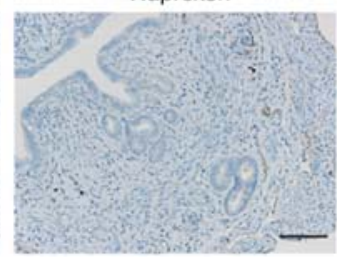

$\mathrm{ADE}$

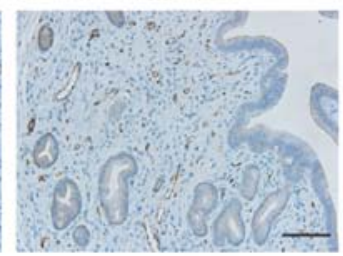

Aspirin

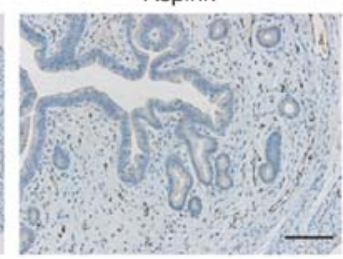

Celecoxib low

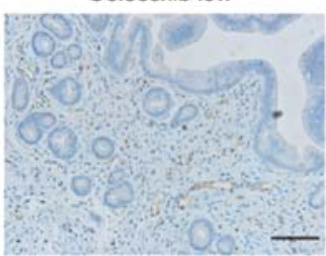

Ibuprofen

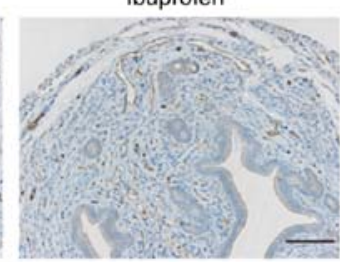

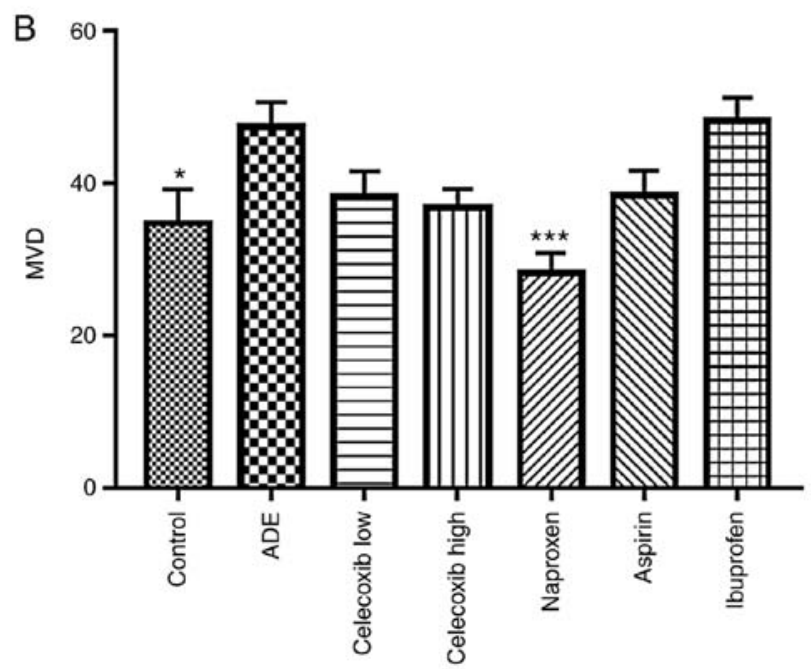

Figure 7. Celecoxib has no effect on vessel density, as evaluated by immunoreactivity staining of CD31. (A) Representative immunohistochemical staining images of CD31 in specimens from mice subjected to non-selective nonsteroidal anti-inflammatory drugs and celecoxib treatment. Scale bars, $100 \mu \mathrm{m}$. (B) Immunohistochemistry quantification of CD31 expression in uteri. ${ }^{* * * *} \mathrm{P}<0.001$ and ${ }^{*} \mathrm{P}<0.05$ vs. ADE. ADE, adenomyosis. MVD, mean vessel density.
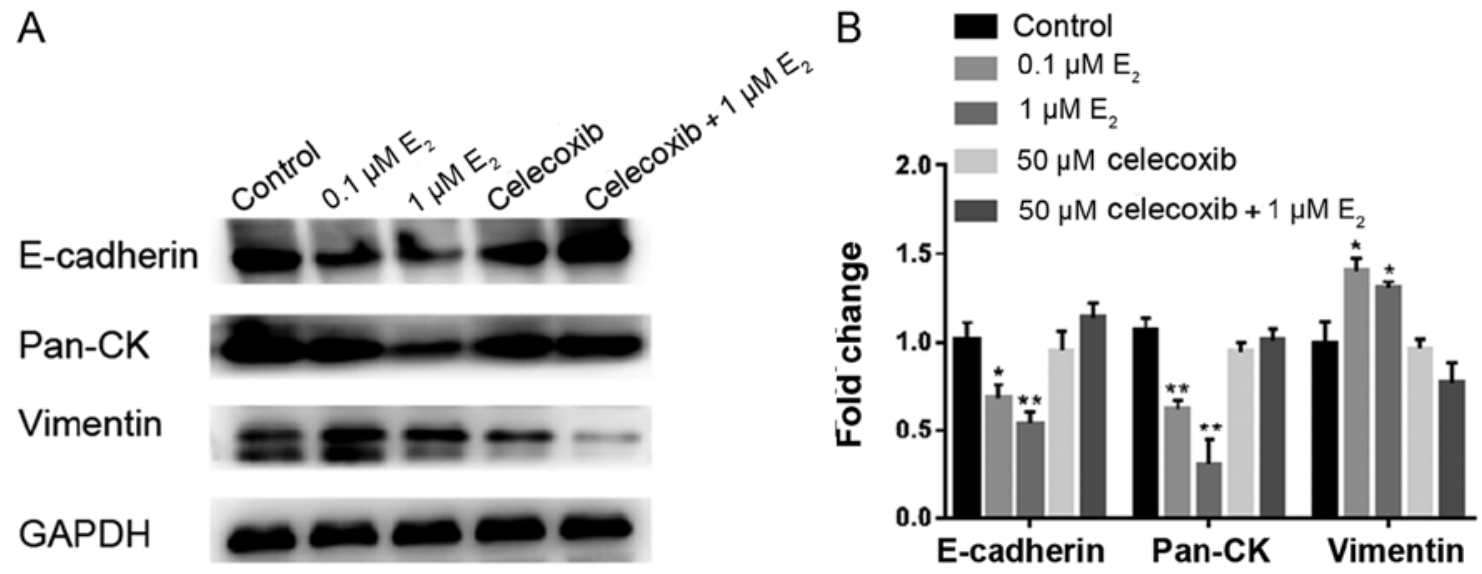

Figure 8. Celecoxib inhibited epithelial-mesenchymal transition in Ishikawa cells following E2 treatment. (A) Representative western blotting images and (B) corresponding quantitative analysis of E-cadherin, Pan-cytokeratin, vimentin expression after treatment with E2 or celecoxib for $48 \mathrm{~h}$. ${ }^{* *} \mathrm{P}<0.01$ and ${ }^{*} \mathrm{P}<0.05$ vs. Control. Pan-CK, Pan-cytokeratin; E2, estradiol.

increased, while that of $\mathrm{N}$-cadherin was decreased. The reversion of EMT was also observed in the nsNSAIDs-treated groups, including aspirin and ibuprofen. Additionally, celecoxib abrogated EMT in response to high concentrations of estrogen in Ishikawa cells, suggesting that COX-2 inhibition may reverse EMT during adenomyosis.

Tissue fibrosis is characterized by the accumulation of extracellular matrix (ECM) and loss of cellular 
homeostasis (49). In adenomyosis, each cyclic bleed accompanied with vascular or tissue injury experienced by the ectopic endometrium is followed by tissue repair (50). On tissue injury, evolutionarily conserved mechanisms initiate the tissue repair process, which is known to involve EMT (51). However, with persistent cycles of tissue injury followed by repair, the physiological wound-healing response can become aberrant, resulting in excessive ECM deposition leading to fibrosis (52). In the present study, fibrosis was also observed in the uteri of mice with ADE, which was reduced by celecoxib treatment, suggesting an important role for COX-2 in fibrotic progression during adenomyosis. Additionally, repression of fibrosis was also observed in mice treated with nsNSAIDs, including aspirin and ibuprofen.

To explore the uterine vascular responses to celecoxib further, the present study also examined vessel density and observed that celecoxib did not exert significant effects on angiogenesis. The interpretation of this finding with regard to the antiangiogenic effects of celecoxib in this model is limited. In view of the angiogenesis effect of the increased expression or activity of Cox-2, recently the inhibition of Cox-2 by celecoxib has been explored to be used in the treatment of diverse cancers such as breast cancer and hepatocellular carcinoma $(18,53,54)$. So further should be performed in larger sample populations or in vitro to reveal the association between the inhibition of Cox-2 by celecoxib and angiogenesis, which may provide a more solid theoretical basis for the application of celecoxib for the treatment of adenomyosis.

In conclusion, nsNSAIDs and the COX-2 selective inhibitor celecoxib markedly reduced the severity of adenomyosis in the present study. In particular, celecoxib demonstrated the greatest efficacy, which may serve as a promising therapeutic intervention strategy due to previous reports of superior tolerance in humans (54). The celecoxib-mediated suppression of adenomyosis is likely to be multifactorial, including estrogen production in the uterus, fibrosis and EMT, which should enable the clinical use of celecoxib to be extended beyond analgesia into adenomyosis. The present study supports further investigation into the use of celecoxib as novel non-hormonal, non-surgical alternative for the treatment of adenomyosis.

\section{Acknowledgements}

The authors would like to thank Dr Zhu Zhiling (Obstetrics and Gynecology Hospital of Fudan University, Shanghai, China) for her technical support.

\section{Funding}

The present study was supported by funding from the National Key R\&D Program of China (grant no. 2016YFC1303100) and the National Natural Science Foundation of China (grant nos. 31570803 and 81773090).

\section{Availability of data and materials}

The datasets used and/or analyzed during the present study are available from the corresponding author on reasonable request.

\section{Authors' contributions}

ZJ performed the lab experiments, analyzed the data and wrote the manuscript. XW contributed to the experimental preparation. HL and CX contributed to the conception of the study and critically revised the manuscript. CX supervised the research project and gave final approval.

\section{Ethics approval and consent to participate}

All experiments were performed under the guidelines of the National Research Council's Guide for the Care and Use of Laboratory Animals, and were approved by the Institutional Experimental Animals Review Board of Shanghai Obstetrics and Gynecology Hospital of Fudan University (Shanghai, China).

\section{Patient consent for publication}

Not applicable.

\section{Competing interests}

The authors declare that they have no competing interests.

\section{References}

1. García-Solares J, Donnez J, Donnez O and Dolmans MM: Pathogenesis of uterine adenomyosis: Invagination or metaplasia? Fertil Steril 109: 371-379, 2018.

2. Bazot $M$ and Daraï E: Role of transvaginal sonography and magnetic resonance imaging in the diagnosis of uterine adenomyosis. Fertil Steril 109: 389-397, 2018.

3. Leyendecker G, Wildt L and Mall G: The pathophysiology of endometriosis and adenomyosis: Tissue injury and repair. Arch Gynecol Obstet 280: 529-538, 2009.

4. Pontis A, D'Alterio MN, Pirarba S, de Angelis C, Tinelli R and Angioni S: Adenomyosis: A systematic review of medical treatment. Gynecol Endocrinol 32: 696-700, 2016.

5. Spitz IM: Clinical utility of progesterone receptor modulators and their effect on the endometrium. Curr Opin Obstet Gynecol 21: 318-24, 2009.

6. Badawy AM, Elnashar AM and Mosbah AA: Aromatase inhibitors or gonadotropin-releasing hormone agonists for the management of uterine adenomyosis: A randomized controlled trial. Acta Obstet Gynecol Scand 91: 489-495, 2012.

7. Fawzy M and Mesbah Y: Comparison of dienogest versus triptorelin acetate in premenopausal women with adenomyosis: A prospective clinical trial. Arch Gynecol Obstet 292: 1267-1271, 2015.

8. Vigano P, Corti L and Berlanda N: Beyond infertility: Obstetrical and postpartum complications associated with endometriosis and adenomyosis. Fertil Steril 104: 802-812, 2015.

9. Huang TS, Chen YJ, Chou TY, Chen CY, Li HY, Huang BS, Tsai HW, Lan HY, Chang CH, Twu NF, et al: Oestrogen-induced angiogenesis promotes adenomyosis by activating the Slug-VEGF axis in endometrial epithelial cells. J Cell Mol Med 18: 1358-1371, 2014.

10. Marjoribanks J, Ayeleke RO, Farquhar C and Proctor M: Nonsteroidal anti-inflammatory drugs for dysmenorrhoea. Cochrane Database Syst Rev: CD001751, 2015.

11. Lethaby A, Duckitt K and Farquhar C: Non-steroidal anti-inflammatory drugs for heavy menstrual bleeding. Cochrane Database Syst Rev: CD000400, 2013.

12. Navone SE, Guarnaccia L, Cordiglieri C, Crisà FM, Caroli M, Locatelli M, Schisano L, Rampini P, Miozzo M, La Verde N, et al: Aspirin affects tumor angiogenesis and sensitizes human glioblastoma endothelial cells to temozolomide, bevacizumab, and sunitinib, impairing vascular endothelial growth factor-related signaling. World Neurosurg 120: e380-e391, 2018. 
13. Zhang L, Li S, Li L, Chen Z and Yang Y: COX2 inhibition in the endothelium induces glucose metabolism normalization and impairs tumor progression. Mol Med Rep 17: 2937-2944, 2018.

14. Hu W, Pei H, Sun F, Li P, Nie J, Li B, Hei TK and Zhou G: Epithelial-mesenchymal transition in non-targeted lung tissues of Kunming mice exposed to X-rays is suppressed by celecoxib. J Radiat Res 59: 583-587, 2018.

15. Gungor H, Ilhan N and Eroksuz H: The effectiveness of cyclooxygenase-2 inhibitors and evaluation of angiogenesis in the mode of experimental colorectal cancer. Biomed Pharmacother 102: 221-229, 2018

16. Sauer CM, Myran DT, Costentin CE, Zwisler G, Safder T, Papatheodorou S and Mucci LA: Effect of long term aspirin use on the incidence of prostate cancer: A systematic review and meta-analysis. Crit Rev Oncol Hematol 132: 66-75, 2018.

17. Gao F, Zafar MI, Jüttner S, Höcker M and Wiedenmann B: Expression and molecular regulation of the Cox 2 gene in gastroenteropancreatic neuroendocrine tumors and antiproliferation of nonsteroidal anti-inflammatory drugs (NSAIDs). Med Sci Monit 24: 8125-8140, 2018

18. Li J, Hao Q, Cao W, Vadgama JV and Wu Y: Celecoxib in breast cancer prevention and therapy. Cancer Manag Res 10: 4653-4667, 2018.

19. Guo CG and Leung WK: Potential strategies in the prevention of nonsteroidal anti-inflammatory drugs-associated adverse effects in the lower gastrointestinal tract. Gut Liver 2019 (Epub ahead of print).

20. Monteiro B and Steagall PV: Antiinflammatory drugs. Vet Clin North Am Small Anim Pract 49: 993-1011, 2019.

21. Melcarne L, García-Iglesias P and Calvet X: Management of NSAID-associated peptic ulcer disease. Expert Rev Gastroenterol Hepatol 10: 723-733, 2016.

22. Granstrom DE: Agricultural (nonbiomedical) animal research outside the laboratory: A review of guidelines for institutional animal care and use committees. ILAR J 44: 206-210, 2003.

23. Shen M, Liu X, Zhang $\mathrm{H}$ and Guo SW: Transforming growth factor $\beta 1$ signaling coincides with epithelial-mesenchymal transition and fibroblast-to-myofibroblast transdifferentiation in the development of adenomyosis in mice. Hum Reprod 31: 355-369, 2016

24. Zhu B, Chen Y, Zhang H, Liu X and Guo SW: Resveratrol reduces myometrial infiltration, uterine hyperactivity, and stress levels and alleviates generalized hyperalgesia in mice with induced adenomyosis. Reprod Sci 22: 1336-1349, 2015.

25. Jin Z, Wang L and Zhu Z: Effect of GuiXiong Xiaoyi Wan in treatment of endometriosis on rats. Evid Based Complement Alternat Med 2015: 208514, 2015.

26. Ding D, Liu X, Duan J and Guo SW: Platelets are an unindicted culprit in the development of endometriosis: Clinical and experimental evidence. Hum Reprod 30: 812-832, 2015.

27. Bird CC, McElin TW and Manalo-Estrella P: The elusive adenomyosis of the uterus-revisited. Am J Obstet Gynecol 112: 583-593, 1972

28. Plone MA, Emerich DF and Lindner MD: Individual differences in the hotplate test and effects of habituation on sensitivity to morphine. Pain 66: 265-270, 1996.

29. Bjarnason I, Scarpignato C, Holmgren E, Olszewski M, Rainsford KD and Lanas A: Mechanisms of damage to the gastrointestinal tract from nonsteroidal anti-inflammatory drugs. Gastroenterology 154: 500-514, 2018.

30. Parente L and Perretti M: Advances in the pathophysiology of constitutive and inducible cyclooxygenases: Two enzymes in the spotlight. Biochem Pharmacol 65: 153-159, 2003.

31. Ota H, Igarashi S, Sasaki $M$ and Tanaka T: Distribution of cyclooxygenase- 2 in eutopic and ectopic endometrium in endometriosis and adenomyosis. Hum Reprod 16: 561-566, 2001.

32. Wang $\mathrm{Y}, \mathrm{Qu} \mathrm{Y}$ and Song W: Genetic variation in COX-2 -1195 and the risk of endometriosis and adenomyosis. Clin Exp Obstet Gynecol 42: 168-172, 2015.

33. Maia HJ Jr, Haddad C, Pinheiro N and Casoy J: The effect of oral contraceptives on aromatase and Cox- 2 expression in the endometrium of patients with idiopathic menorrhagia or adenomyosis. Int J Womens Health 5: 293-299, 2013.

34. Manna PR, Molehin D and Ahmed AU: Dysregulation of aromatase in breast, endometrial, and ovarian cancers: An overview of therapeutic strategies. Prog Mol Biol Transl Sci 144: 487-537, 2016.
35. Chen YJ, Li HY, Huang CH, Twu NF, Yen MS, Wang PH, Chou TY, Liu YN, Chao KC and Yang MH: Oestrogen-induced epithelial-mesenchymal transition of endometrial epithelial cells contributes to the development of adenomyosis. J Pathol 222: 261-270, 2010.

36. Khan KN, Kitajima M, Hiraki K, Fujishita A, Nakashima M and Masuzaki H: Involvement of hepatocyte growth factor-induced epithelial-mesenchymal transition in human adenomyosis. Biol Reprod 92: 35, 2015.

37. Bilyk O, Coatham M, Jewer $M$ and Postovit LM: Epithelial-to-mesenchymal transition in the female reproductive tract: From normal functioning to disease pathology. Front Oncol 7: 145, 2017.

38. Tsui KH, Lee WL, Chen CY, Sheu BC, Yen MS, Chang TC and Wang PH: Medical treatment for adenomyosis and/or adenomyoma. Taiwan J Obstet Gynecol 53: 459-465, 2014.

39. Chamoun-Emanuelli AM, Bryan LK, Cohen ND, Tetrault TL, Szule JA, Barhoumi R and Whitfield-Cargile CM: NSAIDs disrupt intestinal homeostasis by suppressing macroautophagy in intestinal epithelial cells. Sci Rep 9: 14534, 2019.

40. Lands LC and Stanojevic S: Oral non-steroidal anti-inflammatory drug therapy for lung disease in cystic fibrosis. Cochrane Database Syst Rev 9: CD001505, 2019.

41. Vallée A, Lecarpentier Y and Vallée JN: Targeting the canonical WNT/ $\beta$-catenin pathway in cancer treatment using non-steroidal anti-inflammatory drugs. Cells 8: E726, 2019.

42. Rižner TL: The important roles of steroid sulfatase and sulfotransferases in gynecological diseases. Front Pharmacol 7: 30, 2016.

43. Urabe M, Yamamoto T, Kitawaki J, Honjo H and Okada H: Estrogen biosynthesis in human uterine adenomyosis. Acta Endocrinol (Copenh) 121: 259-264, 1989.

44. Benagiano $\mathrm{G}$ and Brosens I: The endometrium in adenomyosis. Womens Health (Lond) 8: 301-312, 2012.

45. Kitawaki J, Noguchi T, Amatsu T, Maeda K, Tsukamoto K, Yamamoto T, Fushiki S, Osawa Y and Honjo H: Expression of aromatase cytochrome $\mathrm{P} 450$ protein and messenger ribonucleic acid in human endometriotic and adenomyotic tissues but not in normal endometrium. Biol Reprod 57: 514-519, 1997.

46. Kitawaki J, Koshiba H, Ishihara H, Kusuki I, Tsukamoto K and Honjo H: Progesterone induction of 17beta-hydroxysteroid dehydrogenase type 2 during the secretory phase occurs in the endometrium of estrogen-dependent benign diseases but not in normal endometrium. J Clin Endocrinol Metab 85: 3292-3296, 2000.

47. Pastushenko I and Blanpain C: EMT transition states during tumor progression and metastasis. Trends Cell Biol 29: 212-226, 2019.

48. Liu X, Shen M, Qi Q, Zhang H and Guo SW: Corroborating evidence for platelet-induced epithelial-mesenchymal transition and fibroblast-to-myofibroblast transdifferentiation in the development of adenomyosis. Hum Reprod 31: 734-749, 2016.

49. Manta L, Suciu N, Toader O, Purcărea RM, Constantin A and Popa F: The etiopathogenesis of uterine fibromatosis. J Med Life 9: 39-45, 2016.

50. Thiery JP, Acloque H, Huang RY and Nieto MA: Epithelial-mesenchymal transitions in development and disease. Cell 139: 871-890, 2009.

51. Hinz B: Formation and function of the myofibroblast during tissue repair. J Invest Dermatol 127: 526-537, 2007.

52. Thannickal VJ, Zhou Y, Gaggar A and Duncan SR: Fibrosis: Ultimate and proximate causes. J Clin Invest 124: 4673-4677, 2014.

53. Tai Y, Zhang LH, Gao JH, Zhao C, Tong H, Ye C, Huang ZY, Liu R and Tang CW: Suppressing growth and invasion of human hepatocellular carcinoma cells by celecoxib through inhibition of cyclooxygenase-2. Cancer Manag Res 11: 2831-2848, 2019.

54. Saxena P, Sharma PK and Purohit P: A journey of celecoxib from pain to cancer. Prostaglandins Other Lipid Mediat 147: 106379, 2019 (Epub ahead of print).

(i) $\Theta$ This work is licensed under a Creative Commons Attribution-NonCommercial-NoDerivatives 4.0 International (CC BY-NC-ND 4.0) License. 